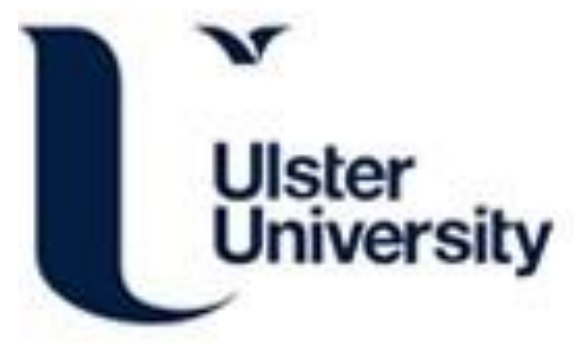

\title{
New Algorithms for Encoding, Learning and Classification of fMRI Data in a Spiking Neural Network Architecture: A Case on Modelling and Understanding of Dynamic Cognitive Processes
}

Kasabov, N., Zhou, L., Doborjeh, M., Doborjeh, Z., \& Yang, J. (2017). New Algorithms for Encoding, Learning and Classification of fMRI Data in a Spiking Neural Network Architecture: A Case on Modelling and

Understanding of Dynamic Cognitive Processes. IEEE Transactions on Cognitive and Developmental Systems, 9(4), 293-303. https://doi.org/10.1109/TCDS.2016.2636291

Link to publication record in Ulster University Research Portal

Published in:

IEEE Transactions on Cognitive and Developmental Systems

Publication Status:

Published (in print/issue): 07/12/2017

DOI:

10.1109/TCDS.2016.2636291

\section{Document Version}

Author Accepted version

\section{General rights}

Copyright for the publications made accessible via Ulster University's Research Portal is retained by the author(s) and / or other copyright owners and it is a condition of accessing these publications that users recognise and abide by the legal requirements associated with these rights.

\section{Take down policy}

The Research Portal is Ulster University's institutional repository that provides access to Ulster's research outputs. Every effort has been made to ensure that content in the Research Portal does not infringe any person's rights, or applicable UK laws. If you discover content in the Research Portal that you believe breaches copyright or violates any law, please contact pure-support@ulster.ac.uk. 


\title{
New Algorithms for Encoding, Learning and Classification of fMRI Data in a Spiking Neural Network Architecture: A Case on Modelling and Understanding of Dynamic Cognitive Processes
}

\author{
Nikola Kasabov, Fellow IEEE, Lei Zhou*, Maryam G. Doborjeh*, Zohreh Gholami, Jie Yang
}

\begin{abstract}
The paper argues that, the third generation of neural networks - the spiking neural networks (SNN), can be used to model dynamic, spatio-temporal, cognitive brain processes measured as functional magnetic resonance imaging (fMRI) data. The paper proposes a novel method based on the NeuCube SNN architecture for which the following new algorithms are introduced: fMRI data encoding into spike sequences; deep unsupervised learning of fMRI data in a 3D SNN reservoir; classification of cognitive states; connectivity visualization and analysis for the purpose of understanding cognitive dynamics. The method is illustrated on two case studies of cognitive data modelling from a benchmark fMRI data set of seeing a picture versus reading a sentence.
\end{abstract}

Index Terms - Spiking neural networks, perceptual dynamics, fMRI data, NeuCube, deep learning in spiking neural networks, brain functional connectivity, classification, neuromorphic cognitive systems.

\section{INTRODUCTION}

The main question that the paper addresses is: Can the third generation of neural networks - spiking neural networks (SNN) be used to model and understand dynamic, spatio-temporal, cognitive processes in the brain? A question to follow would be: Can this approach be translated into intelligent robotic systems?

The paper argues that, SNN can be used to model data that represent brain, spatio-temporal cognitive processes. Such models can be further implemented as neuromorphic cognitive systems using the latest neuromorphic hardware platforms.

The paper proposes new algorithms for encoding, learning and classification of functional magnetic resonance imaging (fMRI) data that measure dynamic cognitive processes. The algorithms are part of the recently proposed NeuCube SNN architecture. The model is illustrated on two case study fMRI data related to seeing a picture versus reading a sentence.

FMRI provides a non-invasive way to collect massive amounts of Spatio-Temporal Brain Data (STBD), providing

\footnotetext{
Nikola Kasabov is with knowledge Engineering and Discovery Research Institute, Auckland University of Technology, Auckland 1010, New Zealand (nkasabov@aut.ac.nz).

* Lei Zhou is with Shanghai Jiao Tong University, Shanghai, China, contact author (minizon@sina.com).
}

insights into brain structures and processes for researchers and clinicians [1] - [3]. Functional MRI uses Blood-Oxygen-Level Dependent (BOLD) contrast to measure brain activity by detecting changes in blood flow. Several analytical methods have been used to analyse these data, such as General Linear Method (GLM) [4]; Principal Component Analysis [5]; Independent Component Analysis [6], [7]; and Temporal Cluster Analysis [8]; but they all have limitations. Various techniques have been developed to analyse the brain's activation, functional connectivity [9], [10] or effective connectivity [11], [12] in fMRI data, but none of these methods can capture the deep spatio-temporal dynamics 'hidden' in the data that represent the dynamics of the cognitive processes. Deep machine learning methods have been developed for traditional neural networks with fixed structures of layers and static input data [13]-[17]. However, brain activity, being consistent in local clusters due to the activation effects [18], must be treated as a dynamic spatio-temporal process [19], [20]. Helpfully, SNN have the ability to learn complex spatiotemporal data [21]-[37], [44]-[47].

The main question that this paper answers is: Can brain cognitive processes, measured as fMRI data, be modelled and understood through deep learning in a SNN architecture?

The algorithms introduced here present an alternative approach to modelling fMRI data with SNN to the method published in [41], even though the two approaches use the same NeuCube SNN architecture [35]. The difference is in the way the dynamical changes in the fMRI data, representing dynamical changes in brain activities, are captured, visualised and interpreted.

\section{SPIKING NEURAL NETWORKS - NEUCUBE}

SNN are computational models that consist of spiking neurons as processing elements, connections between them, and algorithms for learning from data [22]-[33], [44]-[47]. Compared to traditional neuronal networks, SNN can integrate

* Maryam Gholami Doborjeh is with Knowledge Engineering and Discovery Research Institute, Auckland University of Technology, Auckland 1010, New Zealand, contact author (mgholami@aut.ac.nz).

Zohreh Gholami Doborjeh is with knowledge Engineering and Discovery Research Institute, Auckland University of Technology, Auckland 1010, New Zealand.

Jie Yang, is with Shanghai Jiao Tong University, Shanghai, China. 
both spatial and temporal components of data. In addition to the neuronal synaptic state, SNN also integrate the notion of time into their operating model. Therefore, using SNN can lead to an increased level of realism in STBD modelling.

SNN are capable of fast parallel information processing and compact representation of space and time. They can also learn quickly and recognize patterns, deeply 'buried' in STBD [34]. A recent SNN architecture, called NeuCube ("Fig. 1") consists of several modules [35]. An input module encodes the input temporal data sequences into spike sequences. A scalable 3D SNNcube is used to spatially map the input variables (e.g. EEG channels, fMRI voxels) according to their original spatial locations, and then the SNNcube is trained using a learning rule, such as the spike-time dependent plasticity (STDP) learning rule [28], to create and update the connections between spiking neurons. These connections capture spatio-temporal relationships between input variables. Next, an output classification/regression module is trained to classify the spiking activity patterns from the SNNcube into predefined class/output values.

NeuCube has been successfully applied for EEG data and for some fMRI data analysis [35] - [38], [41]. When compared with previous work, the model proposed here offers novel algorithms for encoding, initialization, deep learning, classification and visualization of STBD in the NeuCube SNN architecture, all of them facilitating a better understanding of the dynamics of the cognitive processes captured in the data. It is a generic method, applicable to different types of STBD, including fMRI data, but not restricted to it.

Brain processes are spatio-temporal and that is how they are modelled here. We consider STBD as a set of spatially located sources of data (e.g. fMRI voxels, EEG channels etc.) and the data is measured over time (e.g. seconds, milliseconds).

When using the NeuCube architecture, first a SNNcube (reservoir) is created where each neuron represents the 3D coordinates of an area of the brain according to a given template (e.g. Talairach, MNI). The sources of brain data are mapped correspondingly into spatially allocated neurons of the SNNcube called input neurons, preserving the spatial distribution of these sources in the brain. When the SNNcube is trained on the temporal brain data, it captures spatio-temporal changes in the STBD and learns them as connection weights between neurons that map brain areas. These connections can be analysed to reveal functional connectivity of the brain related to a given task.

The material of the paper presents the following novel algorithms for STBD modelling, in particular - fMRI data, namely:

- Data encoding into spikes;

- Deep unsupervised learning in a 3D SNN cube;

- Classification, visualization and interpretation of the 3D SNN connectivity.

This is illustrated in the paper on two case studies of fMRI data: seeing a picture; reading a sentence. Both case represent typical examples of dynamic cognitive processes.

\section{A NEW AlgorithM FOR ENCODING DYNAMIC STBD INTO SPIKE SEQUENCES}

A continuous input brain data signal is encoded into a spike sequence so that the dynamics of the data is preserved. For a given STBD sequence $\mathrm{S}(t)\left(t \in\left\{t_{0}, t_{1}, \ldots, t_{L}\right\}\right)$, we first define the time $t_{m}$ when the signal reaches its minimum value:

$$
t_{m}=\arg \min _{t} S(t), \quad t \in\left\{t_{0}, t_{1}, \ldots, t_{L}\right\}
$$

The time period from $t_{m}$ to $t_{L}$ (the end time of the signal) is considered further and no spikes will be generated before time $t_{m}$. Based on the initial decrease in the signal, $t_{m}$ is set as the starting time point to capture the changes in the signal during a cognitive task. Let $\mathrm{B}(t)$ denote the baseline for $\mathrm{S}(t)$ at time $t\left(t \in\left[t_{m}, t_{L}\right]\right)$ and $\mathrm{B}\left(t_{m}\right)=\mathrm{S}\left(t_{m}\right)$. If at a time moment $t_{i+1}(m \leq i<L)$, the signal value $\mathrm{S}\left(t_{i+1}\right)$ is above the previous baseline $\mathrm{B}\left(t_{i}\right)$, we encode a spike at time $t_{i+1}$ and the baseline is updated as:

$$
\mathrm{B}\left(t_{i+1}\right)=\alpha \mathrm{S}\left(t_{i+1}\right)+(1-\alpha) \mathrm{B}\left(t_{i}\right)
$$

where $\alpha(\alpha \in[0,1])$ is a parameter to control the signal's contribution to the increase of the baseline. Otherwise, if $\mathrm{S}\left(t_{i+1}\right)$ is below $\mathrm{B}\left(t_{i}\right)$, then no spike is encoded at this time and the baseline is reset as $\mathrm{B}\left(t_{i+1}\right)=\mathrm{S}\left(t_{i+1}\right)$. Successive spikes in the resulting spike sequence reflect the increase of the signal, whilst the absence of a spike means a decrease of the signal ("Fig. 2A").

The proposed method accurately encodes the activation information of continuous temporal data into spike trains. This is important for the following interpretation of the trained SNNcube model, because it enables researchers to better understand brain processes that generate the data. This encoding is also robust to noise. Due to a minimum value threshold which is applied to changes in the signal value, small noise perturbations of the signal are not transformed into spikes. This transformation also accounts for the frequency of changes in the raw signal.

The timing of spikes corresponds with the time of change in the input data. The spike sequence is obtained after the encoding process which represents new input information to the SNN model, where the time unit maybe different from the real time of the data acquisition (machine computation time versus data acquisition time).

\section{A NeW ALGORITHM FOR CONNECTIVITY INITIALIZATION AND DEEP LEARNING IN A SNNCUBE}

After the STBD is encoded into spike trains, the next step is to train a SNNcube (see "Fig.1"), where the spike sequences represent the input data. Input variables are mapped to corresponding spiking neurons in the 3D SNNcube with the same $(x, y, z)$ coordinates. The spike trains are then entered into the SNNcube as whole spatio-temporal patterns (samples) of many time units. A sample representing a labelled sequence of cognitive activity over a certain time period. 


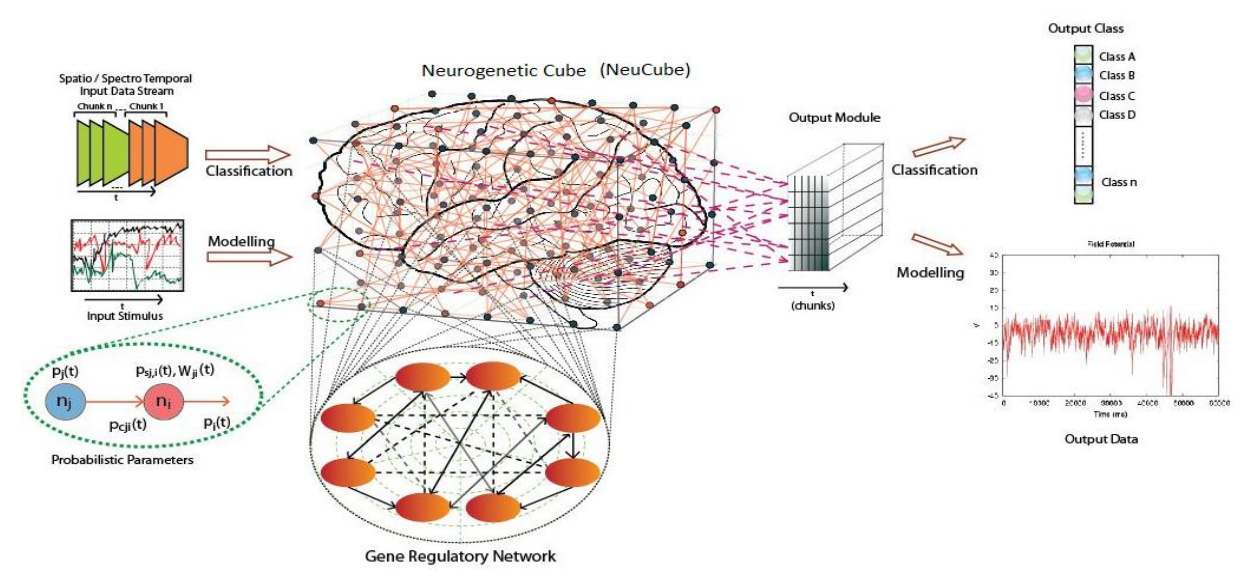

Fig. 1. The NeuCube SNN architecture (from [35]).

Before a learning rule is applied, the connections between spiking neurons in the SNNcube are initialized as follows:

Let $N_{i}$ denote the neighborhood of neuron $i$, defined as:

$$
N_{i}=\left\{j: D_{i j} \leq T, i \neq j\right\},
$$

where $D_{i j}$ denotes the distance between neuron $i$ and neuron $j$, and $\mathrm{T}$ represents the maximum distance allowed for connections between two neurons ( $\mathrm{T}$ is a parameter that is subject to optimization along with other model's parameters). For two neighbouring neurons $i$ and $j$, bidirectional connections are created and connection weights are initialized to zero.

After initializing the connections, the input spike sequences are propagated through the SNNcube and the following learning rule is applied as introduced here: If neuron $i$ and $j$ are connected, and one spike from $i$ precedes that from $j$ within a certain time period, $w_{i j}$ will be increased and $w_{j i}$ left unchanged:

$$
\Delta w_{i j}= \begin{cases}A_{+} \exp \left(\frac{\Delta t}{\tau_{+}}\right) & \text {if } \Delta t \leq 0, \\ 0 & \text { if } \Delta t>0\end{cases}
$$

where $\Delta w_{i j}$ is the synaptic modification (increment of weight); Similar to the STDP parameters as describe in [48], $\Delta t$ is the time difference between spike times of pre-synaptic neuron $i$ and post-synaptic neuron $j$. $\mathrm{A}_{+}$is the maximum quantities of synaptic modification; and $\tau_{+}$represents the time window within which the weight modification is allowed.

After this learning rule is applied to the input data, both bidirectional connection weights are learned, but only the connection with the larger weight of the two bidirectional connections is retained as a final connection between the two neighbouring neurons ("Fig. 2B"). This learning rule is spike time dependent, but different from the STDP rule [28] used in the NeuCube models developed so far [35], [38], [41].
The weaker connection, of the two neuronal connections between neurons $i$ and $j$, is removed and the remaining connection represents a stronger, possible temporal relationship between the two neurons. The removed connection weights are all reset to zero to maintain symmetry of the equation and enable further adaptive training from new data. The trained SNNcube forms a deep architecture as whole spiking input sequences which are learned as chains of connections and spiking activities, regardless of the number of data points measured for every input variable. Unlike hand-crafted layers used in second-generation neural network models [13]- [17], or randomly connected neurons in the computing reservoir of a liquid state machines [22], the chains of directional connections established in the SNNcube represent long spatio-temporal relationships between the sources of the spike sequences (the input variables). Due to the scalable size of a SNNcube, the chains of connected neurons are not restricted in length during learning, which can be considered as unrestricted deep learning, in contrast to existing deep learning methods that use fixed number of layers. As we can see in the following sections, this learning also results in automatic feature extraction, i.e. the automatic selection of a smaller subset of marker input variables.

\section{Feature Selection From a Trained SNN MOdel}

Once the SNNcube is trained with spike sequences of encoded STBD, we can interpret both the connectivity and spiking activity of the model, aiming at new findings about brain functional connectivity and cognitive processes.

A deep chain of connections is learned for each input pattern (sample) in the SNNcube. When entering new input data, the fired chain of neurons and connections will indicate as to which of the previous learned patterns the new one belongs to. This can be used to classify STBD (as shown in the experimental results later in the paper) and for a better understanding of the spatio-temporal brain dynamics. 
A

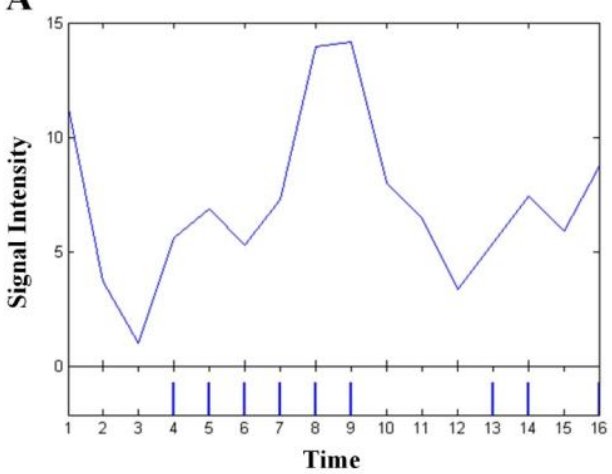

B
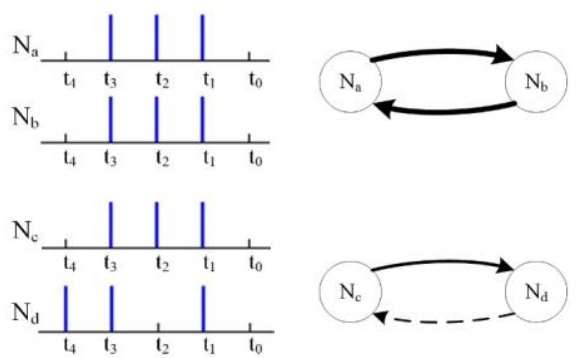

Fig. 2. (A) Spike sequence encoding for one signal. An example of one signal continuous values at 16 time points along with the encoded sequence of spikes (below); The successive spikes from time 4 to 9 represent the increase in the signal, while the absence of spikes from time 10 to 12 means a decrease in the signal; (B) connections established between two connected neurons after unsupervised learning in a SNNcube. Two examples of connection weights established through the proposed method for unsupervised learning between two connected neurons depending on the time of the pre- and post-synaptic spikes of the two neurons. The solid line is the final connection (a thicker line means a larger weight), while the dotted line is removed after learning because of its weaker connection weight. For example, spikes in neuron $N_{c}$ mostly precede those in neuron $N_{d}$, so the learned connection weight $w_{N_{d} N_{c}}$ is smaller, which will be removed after the unsupervised learning.

To analyse the spiking activity of a neuron $i$ in the SNNcube, we define an indicator called activation degree $D_{i}$ :

$$
D_{i}=\frac{\sum_{j}\left(w_{i j}+w_{j i}\right)}{\text { number of neurons in } N_{i}} \quad j \in N_{i}
$$

The parameter $D_{i}$ represents the averaged activation degree of neuron $i$ after a summation of all its inward and outward connection weights. A higher degree of activation of a spiking neuron, represents a greater likelihood that the corresponding loci in the brain are activation foci.

After training the SNNcube, neurons sharing similar spike patterns will have larger weighted connections. This allows us to analyse and understand for example a single subject's response to different stimuli and to compare the responses of different subjects to the same stimulus. A set of spiking neurons with the highest degree of activation representing a given class of stimuli or a cognitive state, will represent a feature set of markers for this class; thus the automatic selection of features as part of the internal deep learning process.

In the following section we illustrate the above model on two case study fMRI data related to cognitive tasks. The SNNcube's parameters used for the two case study experiments are set as: $\alpha=0.5 ; A_{+}=0.1 ; \tau_{+}=1$.

\section{CASE StUdies On Modelling, Classification and FEATURE SELECTION FROM FMRI DATA RELATED TO COGNITIVE TASKS}

We randomly selected two subjects' data from the StarPlus fMRI data related to two cognitive tasks [42]. Our experiments were performed on two subject's data (ID=05680 and $\mathrm{ID}=04820$ ). FMRI data comprised 25 brain regions of interest (ROIs) represented by 5062 and 5015 voxels respectively. For convenience, we will use the terms $\mathrm{ID}=05$ and $\mathrm{ID}=04$ to refer to the above subjects' fMRI data respectively.

The fMRI data was captured every 0.5 seconds (two fMRI volume snapshots per second) while the subjects performed reading a sentence or watching a picture perception tasks during 40 trials. We consider here the first 8 -seconds of recorded data for each trial, during which a 4-second stimulus (picture or sentence) was presented, followed by a 4-second rest. The first 16 volumes of the fMRI data extracted from each trial fell into two classes: watching a picture (Class Pic) or watching a sentence (Class Sen).

As the brain volume has a one-to-one mapping with the SNNcube model, the value of a brain voxel in a brain activation map is defined as the corresponding neuron's activation degree in the SNNcube.

The results from applying the proposed method on fMRI data of subject ID 05 are illustrated in Fig. 3 and Fig. 4.

Brain activation maps for Class Pic and Class Sen were obtained after learning had taken place in the SNNcube ("Fig. $3 \mathrm{Aa}$ "). The neuron's activation degree of the SNNcube was averaged over 20 trials for each class. The voxels in red suggest they were more likely to be activation foci in a certain cognitive state, whilst the blue voxels were less likely to be active. The activation maps were normalized respectively within each class. These maps can be further interpreted, for example, it can be seen from Fig. $3 \mathrm{Bb}$ that when the subject was watching a sentence, the BOLD response in the Calcarine (CALC) region was much stronger than in other regions.

Neurological studies [39], [40] suggest that reading a sentence is more difficult to comprehend than seeing a picture. Therefore, it strongly engages specific regions of the brain along with the visual cortex. The CALC sulcus begins near the occipital lobe, where the primary visual cortex $(V l)$ is concentrated, and passes through the splenium of the corpus callosum, where it is joined at the parieto-occipital sulcus. Our findings confirm that language comprehension, including a reading task, requires more concentration which involves more regions of the brain to act and consequently increases the amount of oxygenated blood required by neurons.

To detect voxel activation, a threshold $T_{D}$ for the neuron's degree of activation and a threshold $T_{w}$ for the neighboring 
neurons' connection weights were defined. The detection procedure is based on the following steps:

Step 1. Find the activation foci in the SNNcube where activation degrees are above $T_{D}$.

Step 2. Set the activation foci as an initial centres of the activation regions $\mathrm{R}$.

Step 3. Expand the activation regions $\mathrm{R}$ in the $\mathrm{SNNcube}$, i.e. add a neuron outside $\mathrm{R}$ if it satisfies the condition that its connection weight with a certain neuron in $\mathrm{R}$ is higher than $T_{w}$.

Step 4. Repeat Step 3 until no neurons outside $\mathrm{R}$ can be included in $\mathrm{R}$. The neurons in $\mathrm{R}$ imply that corresponding voxels in the brain volume are the detected activation voxels.

"Fig. 3B" shows that there are more activated neurons in the CALC region during Class Sen than Class Pic. When the subject was watching a picture, the right hemisphere was slightly more active than the left, but when the subject was reading a sentence, more ROIs in the left hemisphere were involved, including the Left Inferior Parietal lobe (LIPL), Left Superior Parietal Lobe (LSPL), and Left Temporal lobe (LT).
Increased activation of the left cerebral hemisphere is proving to be a more important role for these areas during reading a sentence than during visual object processing. These activations evolved by transferring more spikes between the neurons located in these areas of the SNNcube, reflect more changes in the corresponding voxels' BOLD in the fMRI data.

Since we map voxels to spiking neurons, we can investigate how many activated voxels were involved in multiple brain activities. The percentage $\mathrm{P}$ of overlapped activation voxels is defined as follows:

$$
\mathrm{P}=\frac{R_{P i c} \cap R_{S e n}}{R_{P i c} \cup R_{S e n}}
$$

Where $R_{c}$ denotes the activation voxels in Class c ( c $\in$ $\{$ Pic, Sen $\}$ ). We obtained $P=29.0 \%$ for watching a picture and reading a sentence, indicating that a common part of the brain was engaged in both cognitive states.
$\mathrm{Aa}$

$\mathrm{Ab}$
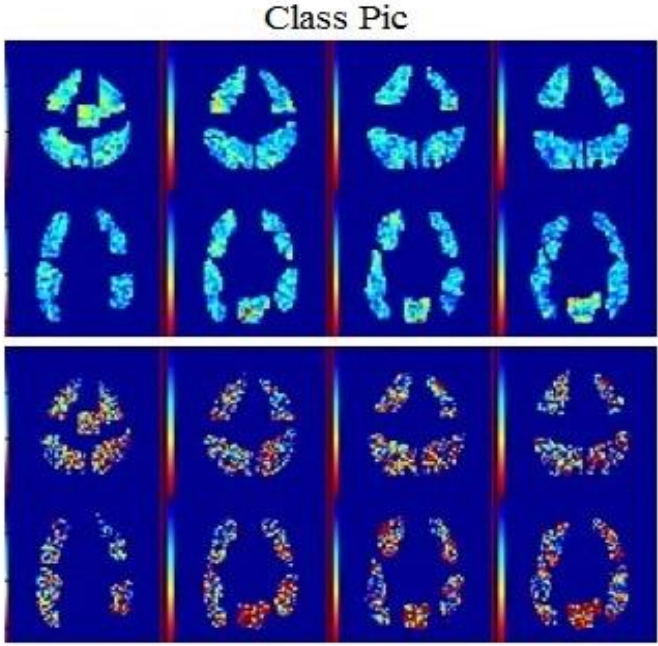

$\mathrm{Ba}$

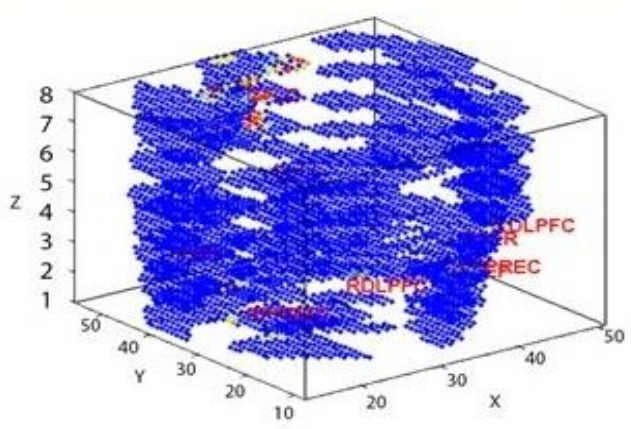

$\mathrm{Bb}$

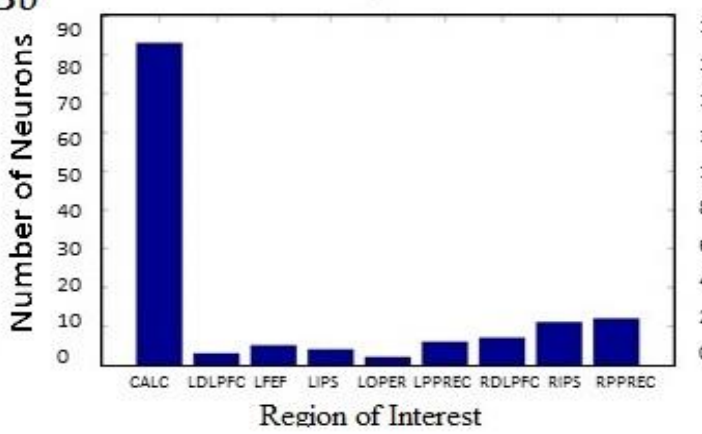

Class Sen
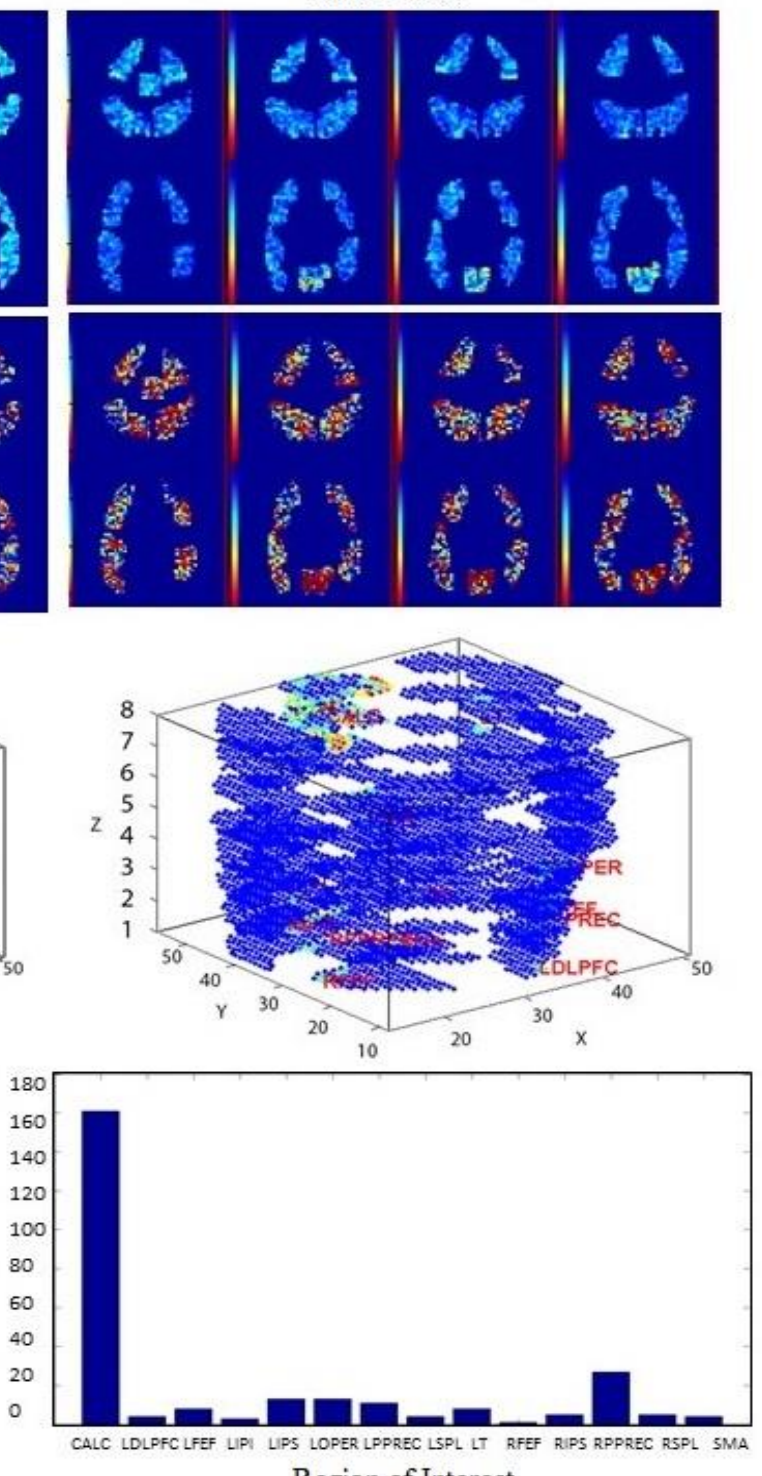

Region of Interest 
C
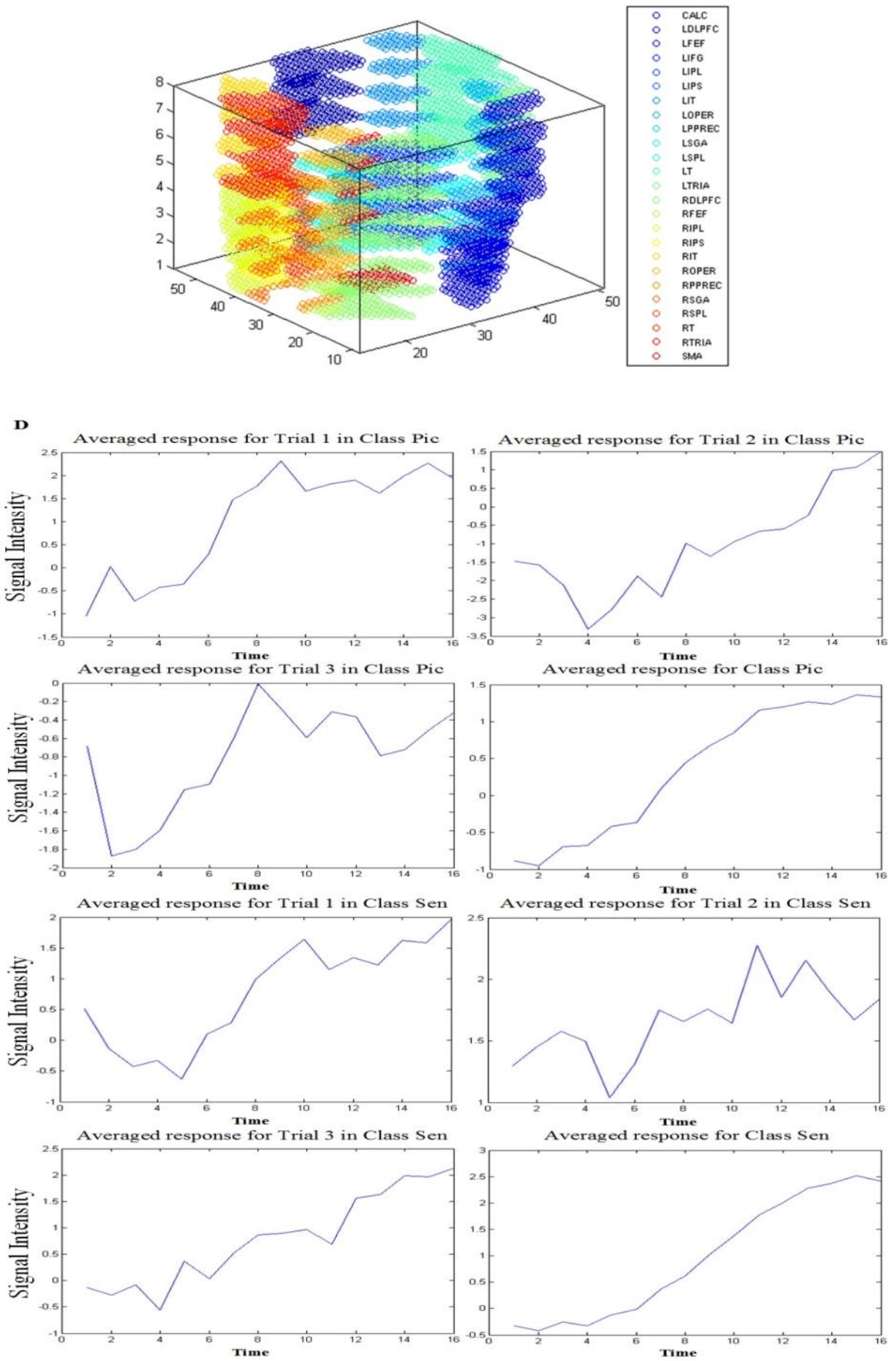

Fig. 3. Brain activation detection and brain regions mapping in the SNNcube for subject ID 05. (Aa) 2D SNNcube activation maps for each class: watching a picture (Class Pic) or reading a sentence (Class Sen); (Ab) Probability map estimated by t-test for Class Pic (left) and Class Sen (right); (Ba) Locations of activation neurons in the averaged SNNcube; (Bb) Histogram of activated neurons with respect to different regions of interest (ROIs) for each class; (C) 25 ROIs were mapped into the SNNcube; (D) Averaged activation of the neurons in the SNNcube from "Fig. 3B" for individual trials for Class Pic and Class Sen. Abbreviations: CALC - calcarine; DLPFC - left dorsolateral prefrontal cortex; FEF - frontal eye fields; IFG - inferior frontal gyrus; IPL - left inferior parietal lobe; IPS - intraparietal sulcus; IT - inferior temporal gyrus; OPER - pars opercularis; PPREC - posterior precentral gyrus; SGA - supramarginal gyrus; SPL - superior parietal lobe; T - temporal lobe; TRIA - pars triangularis; SMA - supplementary motor area.

Analysis of the spiking activity in the SNNcube confirms that BOLD responses differ across trials even of the same class, but the averaged BOLD response for each class corresponds to the hemodynamic response function ("Fig. 3D"). In this figure, the response of the activated voxels (shown in the histogram of activated neurons in Fig.3B) is averaged over 16 fMRI time points and presented for 3 trails per class. We also presented the averaged response of all the trials per class. 
To validate the extracted activated voxels, we conduct the ttests of difference in mean responses of the activated voxels between the rest state and each class. The p-value for class Pic is $3.5622 \mathrm{e}-7$, and $5.3622 \mathrm{e}-22$ for class Sen. Thus, at significance level $99.5 \%$ the responses of such extracted activated voxels are significantly different from the rest state. We also compare the mean responses between class Pic and class Sen averaged over the extracted voxels, and it shows that the mean of the BOLD responses in class Sen is significantly larger than that in class Pic ( $\mathrm{p}=8.0237 \mathrm{e}-8$ using $\mathrm{t}$-test).

During the SNNcube's learning process, the evolution of the neurons' activation degrees was also captured ("Fig. 4A"). The set of neurons with higher activation for one stimulus than another represents a set of features for this stimulus. To demonstrate this concept, we selected two sets of 500 neurons from the SNNcube with highest activation degrees for Class Sen and Class Pic correspondingly ("Fig. 4B").

The directional connections in the SNNcube and identification of the featured neurons allow for further analysis of brain functional activity. Chains of connections for Class Sen and Class Pic are presented in "Fig. 4C". These chains are parts of the whole deep learning architecture.
A
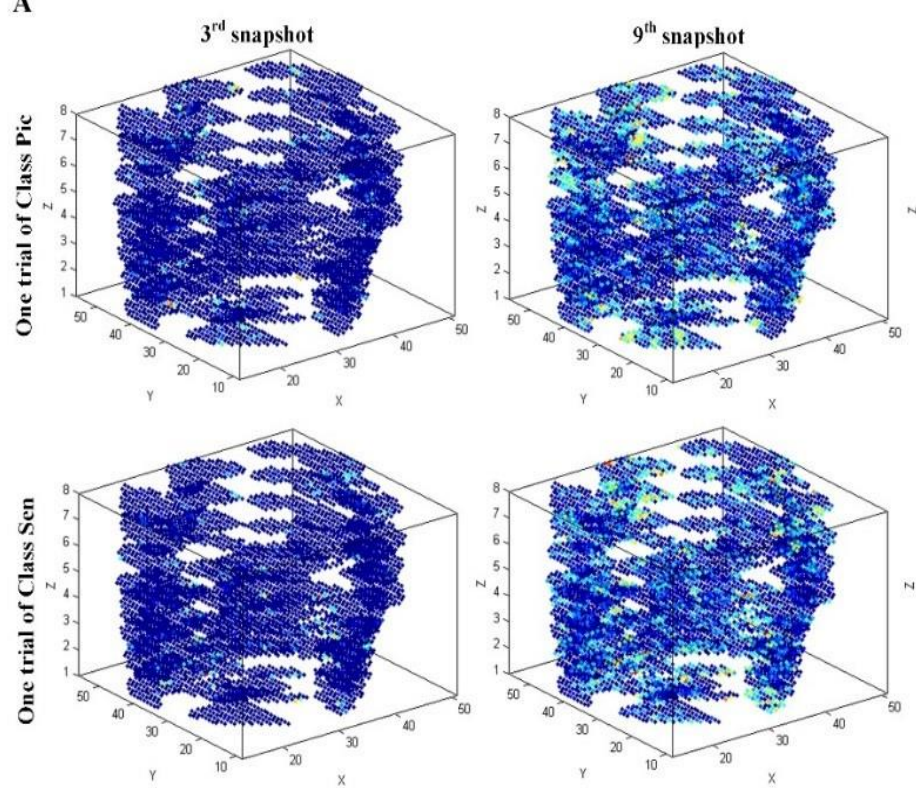

B
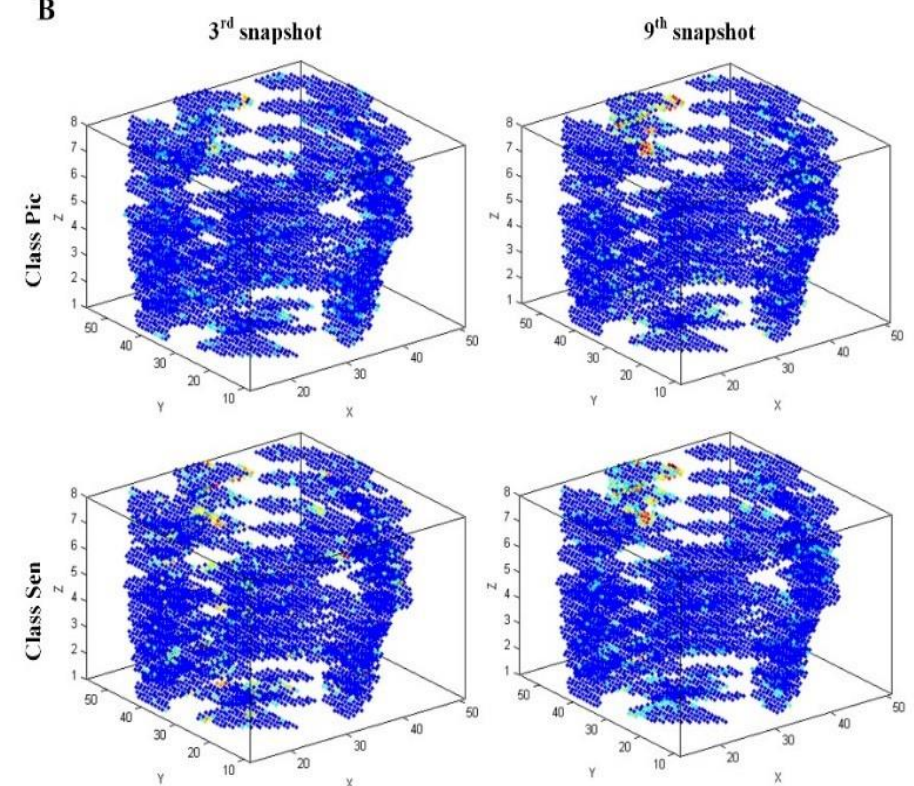

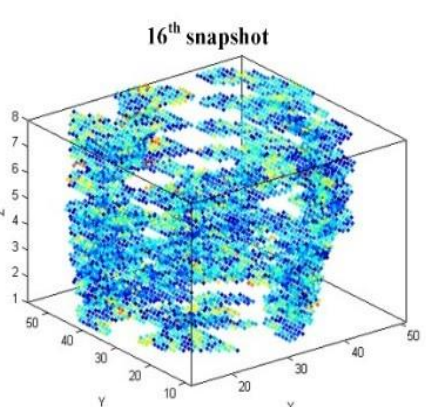

C
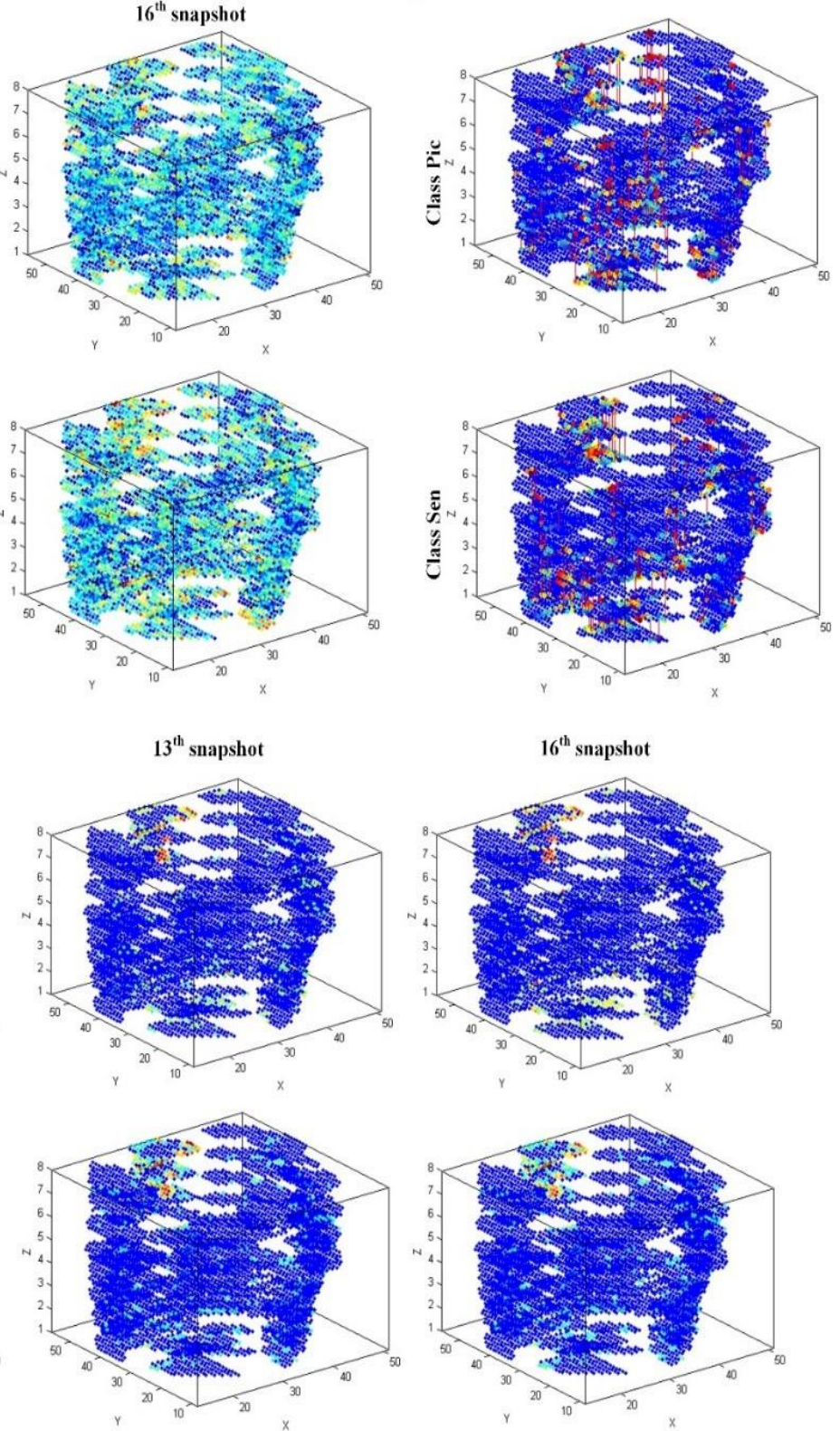

Fig. 4. Evolution of neurons' activation degrees and the deep learning architecture formed in the SNNcube. (A) Neurons' activation degrees at three snapshots when the subject is watching a picture (one trial of Class Pic) or reading a sentence (one trial of Class Sen); The neurons' degrees are normalized at each snapshot for visualization purpose; (B) Locations of neurons with the top 500 activation degrees for Class Pic (upper row) and Class Sen (lower row). These neurons are used as spatio-temporal features for the classification of the two different brain activities; (C) Visualization of typical chains of connections for each class. 
Class Pic

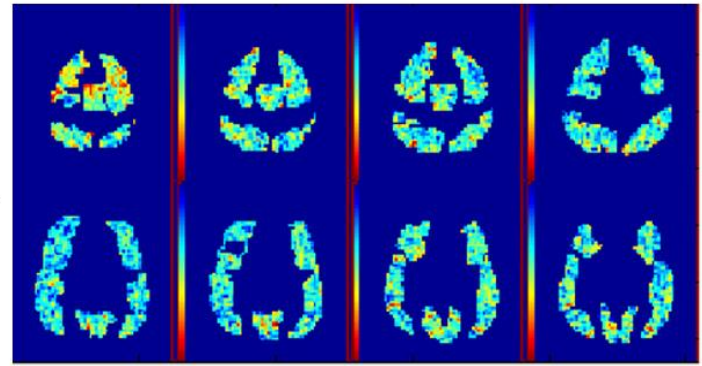

B
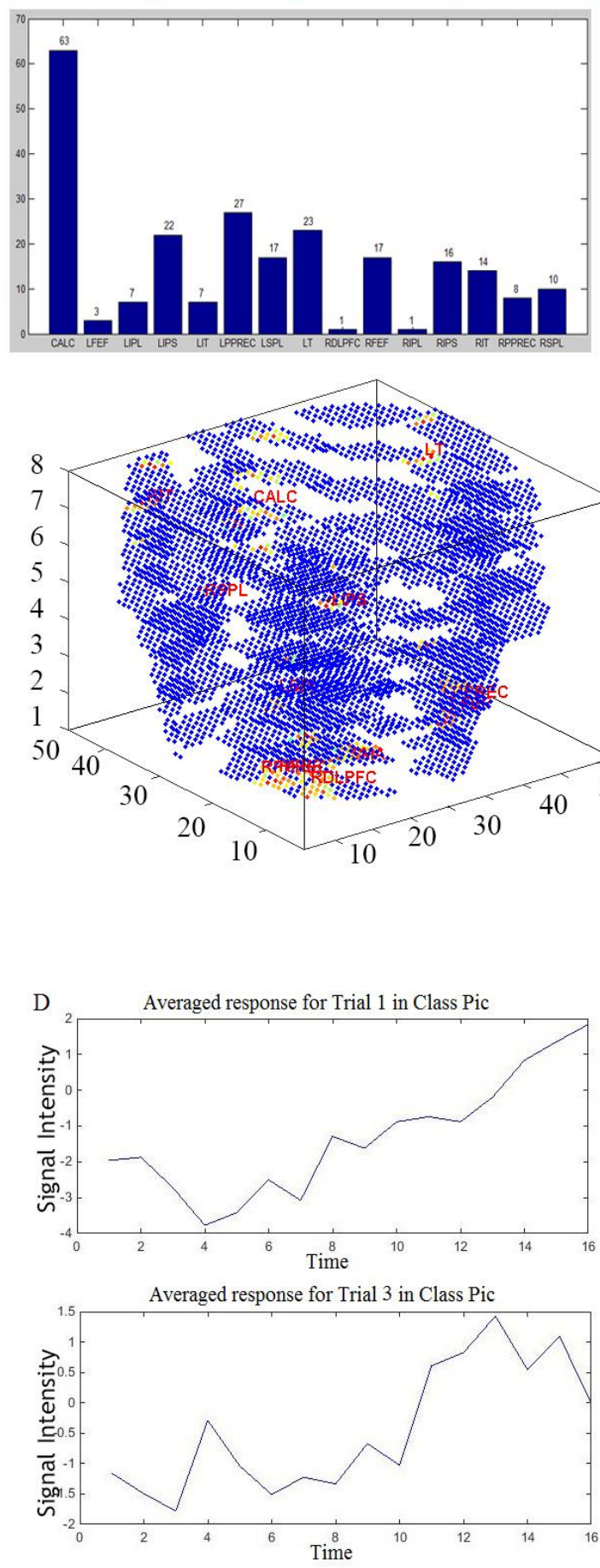

Class Sen
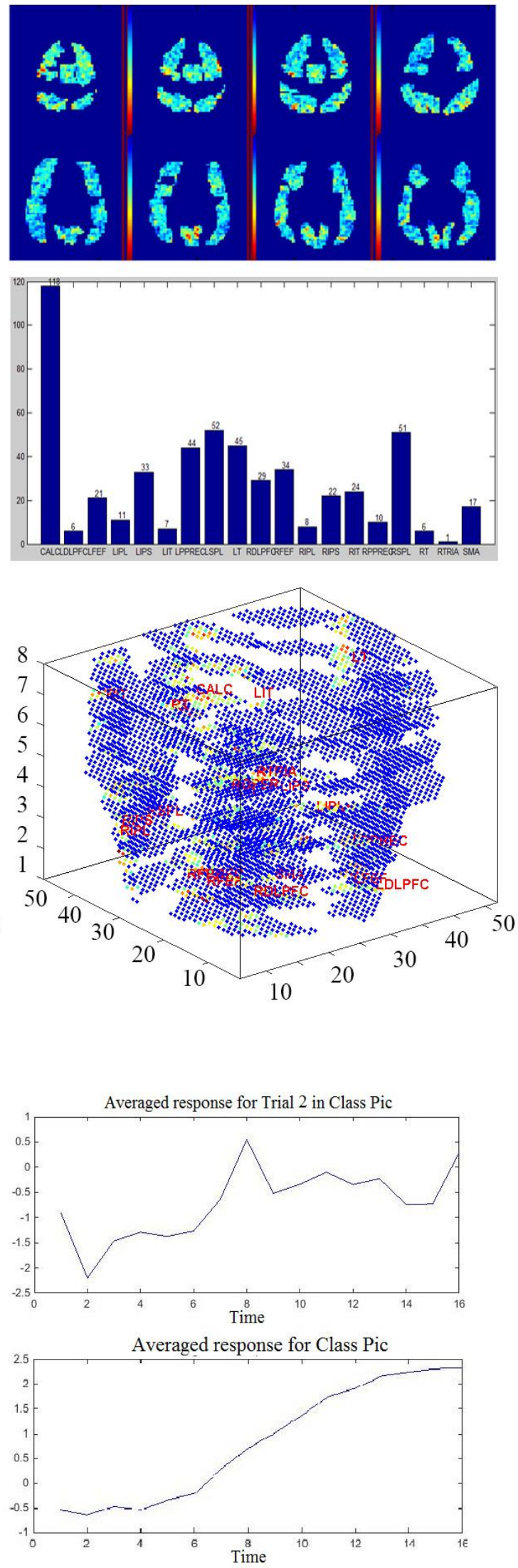

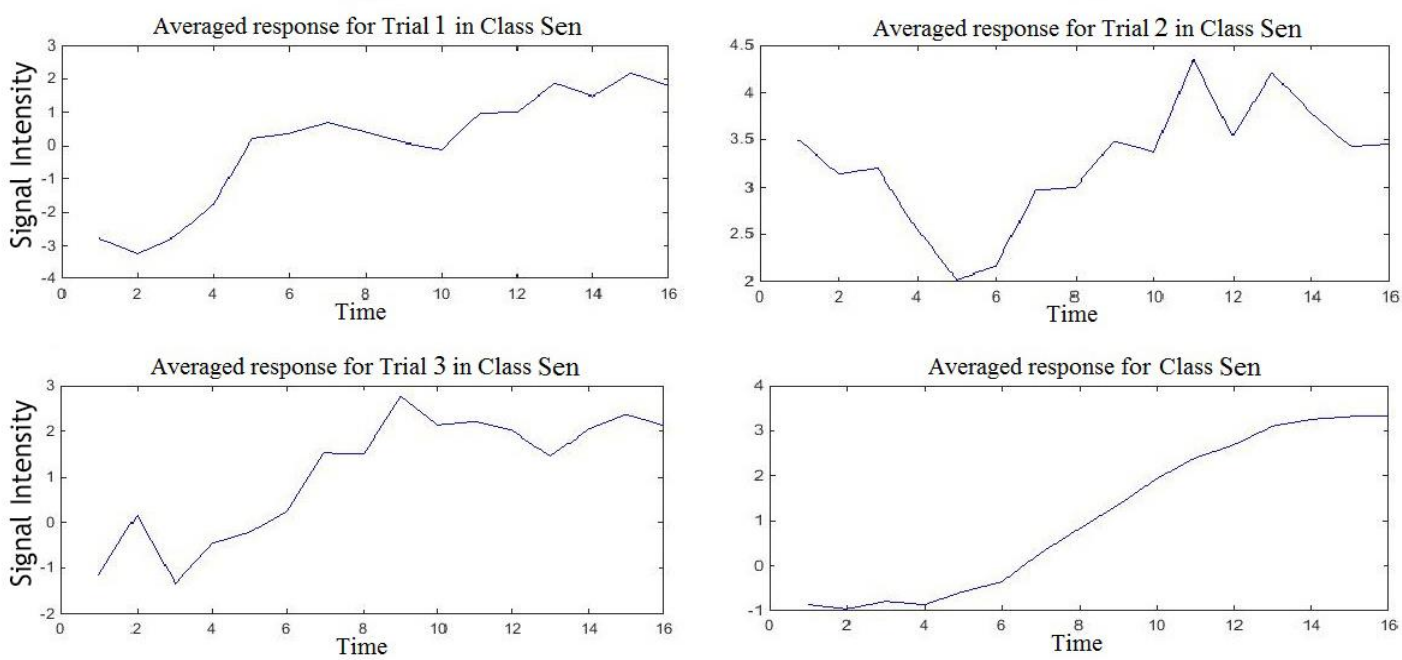

Fig. 5. Brain activation detection is visualized in the SNNcube when trained on ID 04 fMRI data. (A) 2D SNNcube activation maps for class Pic and class Sen; (B) Histogram of activated neurons with respect to different regions of interest (ROIs) for each class; (C) Locations of activation neurons in the averaged SNNcube; (D) Averaged activation of the neurons in the SNNcube for individual trials of Class Pic and Class Sen.

We also applied this methodology to ID: 04 data. The results are illustrated in Fig. 5.

The p-value for class Pic is 5.6203e-4, and $1.8675 \mathrm{e}-8$ for class Sen. It demonstrates that the averaged response of the extracted activated voxels for each class is significantly different from the rest state. The mean of the BOLD responses in class Sen is larger than those in class Pic ( $p=3.2742 \mathrm{e}-4$ using t-test).

We also performed task classification by entering fMRI input data sample by sample, measuring the activity of the featured neurons and classifying the input sample into the class that has a higher number of feature neurons active. The classification accuracy, tested through a leave-one-out cross validation method, is shown in Table. I. The classification accuracy increases over time with more samples entered from each class, because there is a delay in the BOLD response after a stimulus is presented. Once the BOLD response reached a certain level, the difference between watching a picture and reading a sentence became greater (measured as classification accuracy).

\section{TABLE I}

Classification accuracy of class Pic and Sen at each time point of training the SNNcube, calculated using the leave-one-out cross validation method for subjects ID: 05 and 04. There are 20 samples of class Pic and 20 samples of class Sen. The correctly predicted classes are located in the diagonal of the NeuCube confusion table. The classification accuracy improves over time as the SNNcube training process advances.

\begin{tabular}{|c|c|c|c|c|c|c|c|c|c|c|c|c|c|c|c|c|c|c|}
\hline \multicolumn{19}{|c|}{ Subject ID:05 } \\
\hline $\begin{array}{l}\text { Time point of } \\
\text { training the } \\
\text { SNNcube }\end{array}$ & 2 & 3 & 4 & 5 & 6 & 7 & 8 & 9 & 10 & 11 & 12 & 13 & 14 & 15 & 16 & Con & usion & able \\
\hline Class $\quad$ Pic & & & & & & & & & & & & & & & & & Pic & Sen \\
\hline $\begin{array}{l}\text { classification } \\
\text { accuracy in \% }\end{array}$ & 0.0 & 0.15 & 0.1 & 0.2 & 0.4 & 0.65 & 0.7 & 0.65 & 0.75 & 0.7 & 0.8 & 0.8 & 0.85 & 0.8 & 0.9 & Pic & 18 & 2 \\
\hline $\begin{array}{l}\text { Class Sen } \\
\text { classification } \\
\text { accuracy in \% }\end{array}$ & 0.95 & 0.9 & 1 & 0.95 & 0.8 & 0.85 & 0.8 & 0.75 & 0.85 & 0.8 & 0.8 & 0.85 & 0.8 & 0.8 & 0.8 & Sen & 4 & 16 \\
\hline \multicolumn{19}{|c|}{ Subject ID: 04} \\
\hline \multirow{2}{*}{$\begin{array}{l}\text { Class Pic } \\
\text { classification } \\
\text { accuracy in \% }\end{array}$} & \multirow[t]{2}{*}{0.9} & \multirow[t]{2}{*}{0.6} & \multirow[t]{2}{*}{0.4} & \multirow[t]{2}{*}{0.3} & \multirow[t]{2}{*}{0.25} & \multirow[t]{2}{*}{0.35} & \multirow[t]{2}{*}{0.45} & \multirow[t]{2}{*}{0.35} & \multirow[t]{2}{*}{0.3} & \multirow[t]{2}{*}{0.35} & \multirow[t]{2}{*}{0.5} & \multirow[t]{2}{*}{0.55} & \multirow[t]{2}{*}{0.65} & \multirow[t]{2}{*}{0.60} & \multirow[t]{2}{*}{0.65} & & Pic & Sen \\
\hline & & & & & & & & & & & & & & & & Pic & 13 & 7 \\
\hline $\begin{array}{l}\text { Class Sen } \\
\text { classification } \\
\text { accuracy in \% }\end{array}$ & 0.00 & 0.00 & 0.00 & 0.05 & 0.10 & 0.20 & 0.55 & 0.70 & 0.65 & 0.70 & 0.75 & 0.70 & 0.75 & 0.65 & 0.75 & Sen & 5 & 15 \\
\hline
\end{tabular}

\section{CONCLUSION}

In this paper, we proposed a novel methodology for mapping, initialization, deep learning, feature selection, visualization, and classification of STBD using the NeuCube SNN architecture. Feasibility of the proposed methodology has been exemplified here using fMRI data from case studies which include cognitive brain processes.

In the case studies, the 3D SNNcube visualization shows the evolution of neuronal activation degrees and the incrementally 
learned deep patterns from fMRI data. After a SNNcube was trained with spike sequences of encoded fMRI data, we interpreted the model neuronal activation degrees to better understand how this fMRI data relates to the cognitive tasks undertaken by the subjects. Our results show that the NeuCube SNN-based visualization is compatible with the neuroscience literature, which reports that comprehension from reading a sentence is cognitively more complex than watching a picture. Moreover, the NeuCube model reveals the spatio-temporal dynamics of the different cognitive processes.

\section{FUTURE PERSPECTIVES: FROM MODELLING COGNITION WITH SNN TO THE CREATION OF NEUROMORPHIC COGNITIVE AND DEVELOPMENTAL SYSTEMS}

The proposed approach will be further extended in the direction of modelling more complex cognitive data (such as integrated fMRI, EEG, DTI) for a better understanding of brain cognitive processes. Furthermore, we plan to include molecular information such as the level of neurotransmitters, receptors, genes and protein expression as parameters of spiking neurons in the SNNcube [47] (also shown in "Fig.1").

A research question to address in the future is: Based on the results here, which demonstrate that a SNN architecture can be used to model cognitive brain data, can we create neuromorphic cognitive and developmental systems of thousands and millions of spiking neurons? Such systems should be able to manifest complex cognitive behaviour through learning of spatiotemporal patterns. Such systems should be structurally and functionally evolving, i.e. being able to develop new connectivity, new clusters of activities, new output neurons to learn new categories and actions. All these functionalities are enabled in the NeuCube SNN architecture, but the challenge is how to assemble them in an integrated, possibly brain-like, cognitive system, how to allocate input neurons according to input sensory information, how to include available genetic information, how to implement such system on a computer platform, including cloud computing and/or neuromorphic hardware platforms of thousands and millions of neurons [44][47].

\section{ACKNOWLEDGEMENT}

This research was supported by the Knowledge Engineering and Discovery Research Institute of the Auckland University of Technology (AUT) (www.kedri.aut.ac.nz), the Key Laboratory of the Ministry of Education for System Control and Information Processing of Shanghai Jiao Tong University (SJTU) and the Tripartite Project between AUT-SJTU and Xinjiang University in China, funded by Education New Zealand and the Ministry of Education in China. This research is also partly supported by NSFC, China (No: 61572315) and 973 Plan, China (No. 2015CB856004). We thank Anne French for her active role in editing the paper and the reviewers for the constructive suggestions to make this paper much better now.
Supplementary figures and videos related to the paper can be downloaded from http://www.kedri.aut.ac.nz/neucube/fmri. A free copy of a student version of a NeuCube development software system can be obtained from http://www.kedri.aut.ac.nz/neucube/.

\section{REFERENCES}

[1] A. Liu, X. Chen, J. Z. Wang, Q. Xu, S. Appel-Cresswell and M. McKeown, "A genetically informed, group fmri connectivity modeling approach: Application to schizophrenia," IEEE Transactions on Biomedical Engineering, vol. 61, no. 3, pp. 946-956, 2014

[2] P. Rodriguez, M. nderson, V. D. Calhoun and T. Adali, "General Nonunitary Constrained ICA and its Application to Complex-Valued fMRI Data," IEEE Transactions on Biomedical Engineering, vol. 62, no. 3, pp. 922-929, 2015

[3] H. Siegelmann, "How Brain Architecture Leads to Abstract Thought," 2015. [Online]. Available: http://www.umass.edu/newsoffice/article/how-brain-architectureleads-abstract. [Accessed 81 2016]

[4] K. Friston, A. P. Holmes, K. J. Worsley, J. Poline, C. D. Frith and R. S. Frackowiak, "Statistical Parametric Maps in Functional Imaging: A General Linear Approach," Human brain mapping, vol. 2, no. 4, pp. 189-210, 1994

[5] K. Friston, C. Frith, P. Liddle and R. Frackowiak, "Functional connectivity: the principal-component analysis of large (PET) data sets," Journal of cerebral blood flow and metabolism, vol. 13, p. 5, 1993

[6] M. J. McKeown, S. Makeig, G. G. Brown, T.-P. Jung, S. S. Kindermann, A. J. Bell and T. J. Sejnowski, "Analysis of fMRI data by blind separation into independent spatial components," Human Brain Mapping, vol. 6, pp. 160-188,1997

[7] T. Franchin, M. G. Tana, V. Cannata, S. Cerutti and A. M. Bianchi, "Independent Component Analysis of EEG-fMRI data for studying epilepsy and epileptic seizures," in International Conference of the IEEE Engineering in Medicine and Biology Society (EMBC),pp.60116014, DOI: 10.1109/EMBC.2013.6610922, 2013

[8] Y. Liu, J.-H. Gao, H.-L. Liu and P. T. Fox, "The temporal response of the brain after eating revealed by functional MRI," Nature, vol. 405, no. 6790, pp. 1058-1062, 2000

[9] J. Neumann, G. Lohmann, J. Derrfuss and D. Y. Von Cramon, "Metaanalysis of functional imaging data using replicator dynamics," Human brain mapping, vol. 25, no. 1, pp. 165-173, 2005

[10] E. Aggioni, M. G. Tana, F. Arrigoni, C. ucca and A. M. Bianchi, "Constructing fMRI connectivity networks: A whole brain functional parcellation method for node definition," Journal of neuroscience methods, vol. 228, pp. 86-99, 2014

[11] A.Fornito, A.Zalesky, C.Pantelis, E.Bullmore, "Schitzophrenia, neuroimaging and connectomics," NeuroImage, vol.62, pp. 2296-2314, 2012

[12] M. G. Tana, A. M. Bianchi, R. Sclocco, T. Franchin and S. Cerutti, "Parcel-based connectivity analysis of fMRI data for the study of epileptic seizure propagation," Brain topography, vol. 25 , no. 4 , pp. 345-361, 2012

[13] G. E. Hinton and R. R. Salakhutdinov, "Reducing the dimensionality of data with neural networks," Science, vol. 314, no. 5786, pp. 504-507, 2006

[14] G. E. Hinton, "Learning multiple layers of representation," Trends in cognitive sciences, vol. 11, no. 10, pp. 428-434, 2007.

[15] G.E.Hinton, "Learning deep architectures for AI," Foundations and trends in Machine Learning, vol. 2, no. 1, pp. 1-127, 2009

[16] Y. LeCun and Y. Bengio, "Convolutional networks for images, speech, and time series," The handbook of brain theory and neural networks, vol. 3361, no. 10, pp. 1995, 1995

[17] J. Schmidhuber, "Deep learning in neural networks: An overview," Neural Networks, vol. 61, pp. 85-117, 2015 
[18] M. K. Carroll, G. A. Cecchi, I. Rish, R. Garg and A. R. Rao, "Prediction and interpretation of distributed neural activity with sparse models," NeuroImage, vol. 44, no. 1, pp. 112-122, 2009

[19] J. Mourao-Miranda, K. J. Friston and M. Brammer, "Dynamic discrimination analysis: a spatial-temporal SVM," NeuroImage, vol. 36, no. 1, pp. 88-99, 2007

[20] B. Ng and R. Abugharbieh, "Modeling spatiotemporal structure in fMRI brain decoding using generalized sparse classifiers," in International Workshop on Pattern Recognition in NeuroImaging (PRNI), pp. 65-68, DOI: 10.1109/PRNI.2011.10, 2011

[21] M. G. Doborjeh, G. Y. Wang, N. Kasabov, R. Kydd and B. Russell, “A Spiking Neural Network Methodology and System for Learning and Comparative Analysis of EEG Data from Healthy versus Addiction Treated versus Addiction Not Treated Subjects," IEEE Transaction on Biomedical Engineering, vol. 63, no. 99, pp. 1830 - 1841, 2016

[22] E. M. Izhikevich, "Polychronization: computation with spikes," Neural computation, vol. 18, no. 2, pp. 245-282, 2006

[23] W. Maass, T. Natschlager and H. Markram, "Real-time computing without stable states: A new framework for neural computation based on perturbations," Neural computation, vol. 14, no. 11, pp. 2531-2560, 2002

[24] W. Gerstner and W. M. Kistler, "Spiking neuron models: Single neurons, populations, plasticity, Cambridge university press," Cambridge university press, 2002.

[25] S. Thorpe and J. Gautrais, "Rank order coding," Computational Neuroscience, DOI: 10.1007/978-1-4615-4831-7_19, pp. 113-118, 1998

[26] D. Verstraeten, B. Schrauwen, M. d'Haene and D. Stroobandt, "An experimental unification of reservoir computing methods," Neural Networks, vol. 20, no. 3, pp. 391-403, 2007

[27] T. Masquelier, R. Guyonneau and S. J. Thorpe, "Competitive STDPbased spike pattern learning," Neural computation, vol. 21, no. 5, pp. 1259-1276, 2009

[28] T. Masquelier, R. Guyonneau and S. J. Thorpe, "Spike timing dependent plasticity finds the start of repeating patterns in continuous spike trains," PloS one, vol. 3, no. 1, p. e1377, 2008

[29] W. Gerstner, "Time structure of the activity in neural network models," Physical review E, vol. 51, no. 1, p. 738, 1995

[30] E. M. Izhikevich, "Which model to use for cortical spiking neurons?," IEEE transactions on neural networks, vol. 15, no. 5, pp. 1063-1070, 2004

[31] S. M. Bohte and J. N. Kok, "Applications of spiking neural networks," Information Processing Letters, vol. 95, no. 6, pp. 519-520, 2005

[32] R. Brette, M. Rudolph, T. Carnevale, M. Hines, D. Beeman and J. Bower, "Simulation of networks of spiking neurons: a review of tools and strategies," Journal of computational neuroscience, vol. 23, no. 3, pp. 349-398, 2007

[33] S. Song, K. Miller and L. F. Abbott, "Competitive Hebbian learning through spike-timing-dependent synaptic plasticity," Nature neuroscience, vol. 3, no. 9, pp. 919-926, 2000

[34] N. Kasabov, K. Dhoble, N. Nuntalid and G. ndiveri, "Dynamic evolving spiking neural networks for on-line spatio-and spectro-temporal pattern recognition," Neural Networks, vol. 41, pp. 188-201, 2013

[35] N. Kasabov, "NeuCube: A spiking neural network architecture for mapping, learning and understanding of spatio-temporal brain data," Neural Networks, vol. 52, pp. 62-76, 2014

[36] N. Kasabov and E. Capecci, "Spiking neural network methodology for modelling, classification and understanding of EEG spatio-temporal data measuring cognitive processes," Information Sciences, vol. 294, pp. 565-575, 2015.

[37] E. Capecci, N. Kasabov and G. Y.Wang, "Analysis of connectivity in NeuCube spiking neural network models trained on EEG data for the understanding of functional changes in the brain: A case study on opiate dependence treatment," Neural Networks, vol. 68, pp. 62-77, 2015.

[38] M. G. Doborjeh, E. Capecci and N. Kasabov, "Classification and segmentation of fMRI Spatio-Temporal Brain Data with a NeuCube evolving Spiking Neural Network model," in IEEE Symposium on Evolving and Autonomous Learning Systems (EALS), pp. 73-80, 2014

[39] M. Yuasa, K. Saito and N. Mukawa, "Brain activity when reading sentences and emoticons: an fMRI study of verbal and nonverbal communication," Electronics and Communications in Japan, vol. 94, no. 5 , pp. 17-24, 2011

[40] R. K. Christensen, "Negative and affirmative sentences increase activation in different areas in the brain," Journal of Neurolinguistics, vol. 22, no. 1, pp. 1-17, 2009

[41] N. Kasabov, M. Doborjeh, Z. Gholami D, "Mapping, Learning, Visualisation and Classification of fMRI data in evolving SpatioTemporal Data Machines of Spiking Neural Networks," IEEE Transaction on Neural Networks and Learning Systems, accepted, 2016.

[42] M. Just, "StarPlus fMRI data," [Online]. Available: http://www.cs.cmu.edu/afs/cs.cmu.edu/project/theo-81/www/. [Accessed 507 2016]

[43] M. E. Lynall, D. S. Bassett, R. Kerwin, P. J. MvKenna, M. Kitzbichler, U. Muller, E. Bullmore, "Functional connectivity and brain networks in Schizophrenia," The Journal of Neuroscience, vol. 30, no. 28, pp. 9477 9487, 2010

[44] S. B. Furber, F. Galluppi, S. Temple and L. A. Plana, "The SpiNNaker Project," Proceedings of the IEEE, vol. 102, no. 5, pp. 652-665, 2014

[45] P. A. Merolla, J.V. Arhur, R. Alvarez-Icaza, A. S. Cassidy, J. Sawada, F. Akopyan et al, "A million spiking neuron integrated circuit with a scalable communication networks and interface," Science, vol. 345, no. 6197, pp. 668-673, 2014

[46] G. Indiveri, B. Linares-Barranco, T. J. Hamilton, A. Van Schaik, R. Etienne-Cummings, T. Delbruck, S.-C. Liu, P. Dudek, P. H fliger and S. Renaud, "Neuromorphic silicon neuron circuits," Frontiers in neuroscience, vol. 5, pp. 73, pp. 1-23, 2011

[47] N. Kasabov, N. Scott, E. Tu, M. Othman, M. Doborjeh, S. Mark et al, "Evolving Spatio-Temporal Data Machines Based on the NeuCube Neuromorphic Framework: Design Methodology and Selected Applications," Neural Networks, vol. 78, pp. 1-14, 2016

[48] J. Sjöström, W. Gerstner, "Spike-timing dependent plasticity", Frontiers in Synaptic Neuroscience, pp. 35, 2010

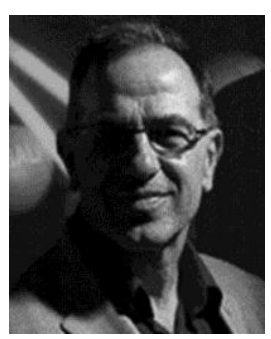

Nikola K. Kasabov $\left(F^{\prime} 10\right)$ received the M.Sc. degree in electrical engineering, and the Ph.D. degree in Mathematical Sciences from Technical University, Sofia, Bulgaria. He is the Director of the Knowledge Engineering and Discovery Research Institute (www.kedri.aut.ac.nz) and the Personal chair of Knowledge Engineering in the School of Engineering, Computer and Mathematical Sciences, Auckland University of Technology, Auckland, New Zealand. He is also an Advisory Professor at Shanghai Jiao Tong University, Shanghai, China, and a Marie Curie Fellow for EvoSpike Project hosted by ETH/UZH Zurich. He has published more than 600 works, among them 180 journal papers and 12 monograph and text books in the area of intelligent system, neural networks, connectionist and hybrid connectionist systems, fuzzy systems, expert systems, bioinformatics, and neuroinformatics. 


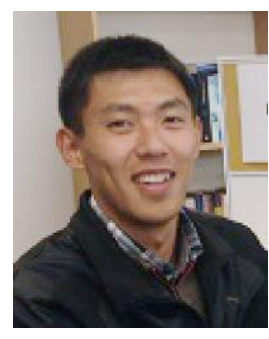

Lei Zhou is a $\mathrm{PhD}$ student at the SJTU and a visiting $\mathrm{PhD}$ student at KEDRI, Auckland University of Technology, New Zealand.

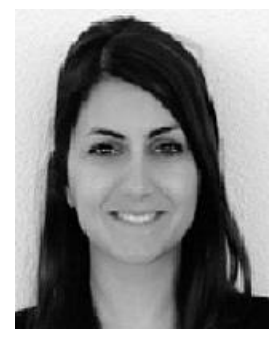

Maryam G. Doborjeh (IEEE, student member 2014) obtained her MSc degree in Computer Science from Easter Mediterranean University, North Cyprus in 2012. She is currently a PhD student at knowledge Engineering and Discovery Research Institute, Auckland University of Technology, New Zealand, under the supervision of Professor Nikola K. Kasabov.

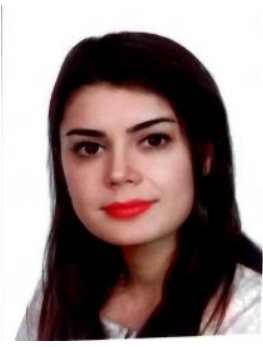

Zohreh Gholami D (IEEE, student member 2016) obtained her a MS. degree (as honor student) in psychology, from Ferdowsi University of Mashhad, Iran, in 2013. She has previous research experiences in Neuromarketing, Cognitive task designing, and EEG and ERP data analysis. She is currently a PhD student and research assistant at KEDRI, Auckland University of Technology, New Zealand.

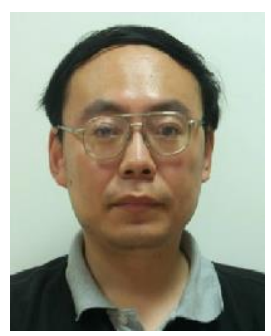

Jie Yang received his Ph.D. degree at Department of Computer Science, University of Hamburg, Germany In 1994. Now he is the Professor and Director of Institute of Image Processing and Pattern recognition in Shanghai Jiao Tong University. $\mathrm{He}$ is the principal investigator of more than 30 national and ministry scientific research projects in image processing, pattern recognition, data mining, and artificial intelligence, including two national 973 research plan projects, three national 863 research plan projects, four National Nature Foundation projects, six international cooperative projects with France, Sweden, Korea, Japan, New Zealand. He has published 5 books and more than 500 articles in national or international academic journals and conferences. 